Relations industrielles

Industrial Relations

\title{
Murdochville: Les faits
}

\section{Roger Chartier}

Volume 12, numéro 4, octobre 1957

URI : https://id.erudit.org/iderudit/1022514ar

DOI : https://doi.org/10.7202/1022514ar

Aller au sommaire du numéro

Éditeur(s)

Département des relations industrielles de l’Université Laval

ISSN

0034-379X (imprimé)

1703-8138 (numérique)

Découvrir la revue

Citer ce document

Chartier, R. (1957). Murdochville: Les faits. Relations industrielles / Industrial

Relations, 12(4), 374-381. https://doi.org/10.7202/1022514ar

Tous droits réservés @ Département des relations industrielles de l’Université Laval, 1957

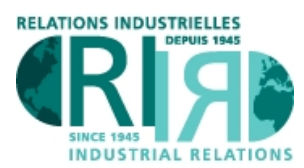

INDUSTRIAL RELATIONS
Ce document est protégé par la loi sur le droit d'auteur. L’utilisation des services d'Érudit (y compris la reproduction) est assujettie à sa politique d'utilisation que vous pouvez consulter en ligne.

https://apropos.erudit.org/fr/usagers/politique-dutilisation/ 


\section{COMMENTAIRES}

\section{Murdochville: Les faits}

\section{Roger Chartier}

\section{INTRODUCTION}

Ce qui suit veut être la séquence objective, la nomenclature en vrac des faits les plus importants qui constituent proprement "l'affaire de Murdochville», dont des chapitres importants viennent de se clore. On ne proposera aucune interprétation systématique de ces faits à la lumière de données sociales, économiques, morales ou juridiques. On n'ignore pas, cependant, que les faits brutaux ont leur éloquence, et que le chroniqueur, par le choix même qu'il effectue des événements historiques, porte constamment des jugements de valeur, fût-ce en toute inconscience. On passe assez vite sur les «violences》 malheureuses de Murdochville, qui sont bien connues du public et qui découlent comme fatalement des prémisses qui suivent.

\section{LA PRÉHISTOIRE}

Dès 1921, M. J.Y. Murdoch, président de la Noranda Mines, s'intéresse au rapport d'un prospecteur selon lequel la péninsule gaspésienne recèlerait en son sein d'importants gisements de cuivre à teneur relativement faible. Une vingtaine d'années s'écoulent, au cours desquelles les recherches en ce sens sont plutôt infructueuses. Il faudra attendre la fin de la seconde guerre mondiale pour que de nouvelles prospections indiquent la présence, à quelque 60 milles à l'ouest de Gaspé (canton de Holland, district de Gaspé), d'environ 65 millions de tonnes de minerai de cuivre. Fidèle à sa promesse, le gouvernement provincial relie le camp Murdoch, nouvellement établi, à la grande route de ceinture gaspésienne par une voie d'accès de premier ordre. En 1952, c'est le percement d'un tunnel dans les flancs du mont Needle, l'aménagement graduel et vigoureusement poussé de la mine et le recrutement chez les pêcheurs et les travailleurs en forêt de la péninsule, mais également chez ces Gaspésiens, nombreux, qui reviennent riches d'une expérience précieuse et prolongée des mines du nord-ouest québecois. La même année, le camp Murdoch devient une municipalité constituée, qui a nom Murdochville; sa population, qui augmente rapidement, aura tôt fait d'atteindre les 2,500 habitants dès 1955 , année où la mine enfin fonctionne à plein rendement avec un personnel d'environ 1,000 hommes. La compagnie Gaspe Copper Mines (une filiale de la Noranda Mines qui en détient $94 \%$ des actions et partage avec elle «sa principale place d'affaires à Noranda 》) s'affaire à la construction de centaines de maisons et d'aménagements communaux élaborés. Ses usines valent au bas mot $\$ 45,000,000$, et sa production mensuelle normale sera de 3,000 tonnes de cuivre.

\section{SYNDICALISME EMBRYONNAIRE}

A l'automne de 1952, l'Union des métallurgistes unis d'Amérique (United Steelworkers of America), local 4881, affiliée au Congrès canadien du travail (COI.), est en pleine phase d'organisation syndicale. Elle se réclame bientôt de quelque $80 \%$ des travailleurs syndicables qui ont signé leur carte d'adhésion et payé leur cotisation. Elle adresse alors à la Commission de relations ouvrières (CRO) une requête en certification. Dans ses représentations à l'encontre de cette demande, la compagnie allègue qu'à cette période les opérations n'en sont pas encore au stade de la production proprement dite, et donc que le groupe des signataires est fort différent du personnel que normalement elle emploiera par la 
suite. La CRO se rend à cet argument et refuse d'accorder le certificat demandé, sans contestation formelle de la part des métallos. Peu de temps après, la compagnie cesse d'avoir besoin des services du président et du secrétaire d'un syndicat bien réel, mais non reconnu.

L’année suivante (1953), les Steelworkers sont de nouveau à l'oeuvre; mais cette fois ils doivent lutter contre l'Union internationale des employés de mines (The International Union of Mine Employees), district de Gaspé, local 544, affiliée directement à une centrale rivale, le Congrès des métiers et du travail du Canada. La compagnie semble pencher du côté de cette dernière union, car à l'hiver de 1953-54, les organisateurs des Steelworkers sont chassés par la police des camps de la compagnie et vont s'établir sous la tente, à quelques milles de la ville; c'est là qu'ils organisent une assemblée de mineurs, tôt dispersée par la police de la compagnie. Au printemps de 1954, les employés de mines de l'union rivale sont certifiés par la CRO et, à la suite de négociations collectives, signent le 16 juillet 1954 avec la compagnie une convention collective renouvelable le 15 juillet 1956.

\section{L'EXPÉRIENCE DE LA VIE À DEUX}

Au dire de la compagnie (dans une requête en vue d'obtenir contre la CRO un bref de prohibition sur lequel on reviendra plus loin), elle « a toujours entretenu avec ses employés, et particulièrement depuis cette convention, les relations les plus harmonieuses 》 ( $(18)$. Le syndicat, qui gagne en maturité au cours de ces années de vie commune, semble pourtant se sentir quelque peu à l'étroit dans un contrat collectif dont une clause en particulier, celle des «droits de la gérance », a un caractère plutôt rébarbatif. Quoi qu'il en soit, les négociations sur les salaires prévues pour janvier 1956 sont assez tendues, et les revendications syndicales, peu goûtées de la compagnie. Le syndicat demande alors les services d'un conciliateur, qui ne vient pas à cause du caractère «prématuré » de la requête syndicale.

Mais voici que se produit un événement important dans le monde syndical canadien: en avril 1956, on assiste à la fusion, au pays, des deux grandes centrales syndicales en cause, le CCT des métallos malheureux et le CMTC des employés de mines en place à Murdochville. Cette fusion suit celle, aux Etats-Unis, du COI et de la FAT en décembre 1955. On permet alors aux mineurs de la Gaspe Copper de déterminer eux-mêmes quel syndicat les représentera: il ne s'agit donc que d'un changement possible d'allégeance syndicale par un groupe d'employés qui demeurera de toute façon sensiblement le même.

Le ler juin 1956, les organisateurs des Steelworkers peuvent chanter victoire, car sur un total de 940 travailleurs syndicables, ils croient détenir 747 cartes d'adhésion avec cotisations en règle, plus 150 cartes signées avec cotisations à venir (soit $80 \%$ ou $95 \%$ des membres de l'unité de négociation). Aussi s'empressentils de demander à la CRO le transfert du certificat de reconnaissance en faveur du groupement à nouvelle affiliation. Les enquêteurs de la CRO se déclarent satisfaits des documents fournis par le syndicat et présentent un rapport favorable à l'émission du nouveau certificat. La compagnie, prévenue comme il se doit par la CRO en fin de juin, réclame à la Commission, le 27 du même mois, un délai jusqu'au 20 juillet, ne se satisfaisant pas des sept jours prévus par la Loi des relations ouvrières pour produire ses représentations à l'encontre de l'émission du certificat. Le 6 juillet, elle exige «communication de la requête en reconnaissance syndicale, de la résolution du groupement relative à cette requête, de la constitution du groupement demandant la certification, et d'une façon générale des pièces produites à l'appui de cette requête en reconnaissance syndicale, soulignant spécialement le droit de la requérante de prendre connaissance des procédures faites et des pièces invoquées contre elle » (requête en vue d'obtenir un bref de prohibition, §5). 
Le 21 juillet 1956, la compagnie reçoit de la CRO des copies, sans noms de signataires ni certificats d'authenticité - car c'est une règle administrative de la Commission de ne pas dévoiler les noms des syndiqués ou des chefs syndicaux en cause - , de la requête en certification et de la résolution de l'assemblée syndicale relative à la demande de certification.

\section{LE BREF DE PROHIBITION CONTRE LA CRO}

Enfin, le ler août 1956, c'est la bombe: une requête du procureur de la Gaspe Copper Mines à la Cour Supérieure pour l'émission d'un bref de prohibition contre la CRO, le syndicat des métallos se trouvant mis en cause. La compagnie, dans cette requête citée plus haut et dont le texte intégral se retrouve dans la revue Relations Industrielles (vol. 12, no 3, juillet 1957, pp. 253-57), allègue que la CRO « refuse injustement, illégalement et sans juridiction de communiquer... tous (ces) renseignements devant obligatoirement apparaître et apparaissant à l'original de la requête (syndicale)...» (\$9).

Et la requête de la compagnie de poursuivre:

\& 12.-C'est volontairement que l'intimée (CRO) entoure de mystère le groupement mis en cause, contre la légalité duquel pourtant la requérante est invitée à faire ses représentations, groupement qui n'a même pas de personnalité légale et qui ne pourrait, à toutes fins pratiques, si tant est qu'il existe, être identifié que par ses soi-disant officiers et ses soi-disant membres...

\& 14.-...l'intimée (CRO) a refusé et refuse de donner communication à votre requérante des pièces produites à l'appui de la requête, et plus particulièrement et surtout des prétendues déclarations ou cartes d'adhésion des employés de la requérante au soi-disant groupement mis en cause, pièces qui sont essentielles à la considération du litige...

«25.-...la requérante est appelée à se défendre contre un groupement mystérieux, insaisissable...

《26.-C'est un droit élémentaire de votre requérante de connaître les personnes qui réclament contre elle la reconnaissance de certains droits, et de sa part, en leur faveur, l'exécution de prétendues obligations...

«30.-Contrairement à tous les usages et à toutes les règles de nos procédures judiciaires, contrairement au droit naturel et constitutionnel de toute partie à un litige, illégalement et sans juridiction, commettant par là un déni de justice flagrant, l'intimée (CRO) entend considérer la demande du groupement mis en cause et se prononcer dans le litige qui l'oppose à votre requérante sans permettre à celle-ci d'être utilement entendue...

«32.-Le bref de prohibition est le seul remède approprié, avantageux et efficace dans les circonstances... »

Le lendemain, 2 août, sans entendre la CRO, un juge de la Cour Supérieure signe le bref introductif d'instance en prohibition, ordonnant à la CRO et au syndicat «de surseoir à toutes procédures dans le litige décrit à la requête présentée en cette cause... en attendant qu'il soit adjugé au mérite... 》 (Cour Supérieure, District de Québec, no 82-558). Les relations strictement légales entre le syndicat et la compagnie se trouvent ainsi interrompues jusqu'au jugement de la Cour Supérieure, et le syndicat, privé d'un certificat auquel il a décidément droit, voit son action professionnelle bloquée totalement, à moins d'un recours à l'«illégalité technique » ou de l'acceptation pratique par la compagnie de l'article 18 de la Loi des relations ouvrières qui dit que 
Rien dans la présente loi n'empêche une association non reconnue de conclure une convention collective; mais une convention ainsi conclue est non avenue le jour où une autre association est reconnue par la Commission pour le groupe que représente cette dernière association.

A l'été de 1956, certains chefs syndicaux se plaignent d'une réduction de grade et de salaire. A la fin de septembre, un vote indique que les nombreux travailleurs présents à une assemblée du syndicat sont favorables, à $98 \%$ près, à la grève. Ce vote vient appuyer le comité syndical de négociation collective, composé de 14 membres, qui s'efforce de discuter de problèmes communs avec la cornpagnie. A partir de ce moment, les chefs syndicaux n'ont pas la partie facile. Au début d'octobre 1956, une lettre est adressée à la compagnie pour l'inviter à négocier une convention. Avant d'exprimer son refus par écrit, sous prétexte que les procédures judiciaires n'ont pas encore abouti, la compagnie accorde des augmentations horaires variant de .07 cts à .20 cts, pendant que les négociations se poursuivent à Noranda entre les Steelworkers et la compagnie-mère, la Noranda Mines. La situation reste tendue: les griefs s'accumulent sans trouver d'exutoire.

\section{LE GRAND COUP: LA GRĖVE}

Le 8 mars 1957, la compagnie pose un geste qui devait se révéler décisif: elle met à pied M. Théo Gagné, maître-plombier à son service depuis le 11 octobre 1954, un employé d'entretien qui avait été prêté à un autre département pour accomplir des travaux de construction. Gagné est \&l'un de plusieurs employés, non pas congédiés, mais temporairement mis à pied le 8 mars, au moment où les travaux auxquels ils étaient alors assignés se sont trouvés complétés. Il devait effectivement rencontrer le gérant de la mine le 11 mars, mais ne se présenta pas au rendez-vous. Dans l'intervalle et sans qu'aucune représentation quelconque ne soit faite à la compagnie, la grève commençait» (Communiqué de la compagnie paru dans La Presse du 8 mai 1957 et daté de la veille). Or, il est fort utile de le mentionner, M. Gagné était -également.président du syndicat des. métallos!

Le dimanche soir, 10 mars, la grève immobilise totalement les aménagements de la Gaspe Copper à Murdochville. Les travailleurs ont interprété le licenciement du chef syndical comme un geste hostile et insultant. Un piquetage efficace s'organise autour de la mine. Une semaine après, le 18 mars, la compagnie présente une requête pour l'émission d'un bref d'injonction intérimaire, valable jusqu'au 3 varil et suivi au besoin d'une ordonnance interlocutoire et permanente. Lo même jour, un juge de la Cour Supérieure donne droit à la requête de la compagnie, interdisant le piquetage («nul et illégal »); il endosse ainsi la position do la compagnie selon laquelle le piquetage est illégal parce que la grève est illégale, le syndicat ne possédant pas de certificat. La requête parle encore de violation du droit de propriété, d'obstruction, de menaces et de dommages. Le lendemain, 19 mars, la compagnie prend action en dommages au montant de $\$ 45,000$ contre quelques chefs syndicaux. Le 2 avril, l'injonction interlocutoire est émise à l'égard du piquetage. Vingt-sept policiers provinciaux arrivent sur les lieux. Les grévistes n'en continuent pas moins à maintenir le piquetage, paisiblement, sans bloquer l'accès à la mine. Le 11 du même mois, le syndicat des Steelworkers à Noranda cède devant une offre intéressante de la compagnie-mère et abandonne en quelque sorte à leur sort les grévistes de Murdochville.

Le 25 avril 1957, cinquante policiers provinciaux viennent prêter main-forte à leurs confrères déjà sur les lieux depuis trois semaines. On venait de découvrir près de l'entrée de la mine une douzaine de bâtons de dynamite. Ainsi s'exprima sur ce point le président du syndicat: "Rien ne justifie la présence de ces policiers, si ce n'est que l'on vent provoquer les grévistes, les entraîner dans des troubles graves, pour ensuite les faire passer pour des agitateurs aux yeux de l'opinion publique 》 (Le Devoir, 26 avril 1957).

Jamais paroles ne furent plus vraies, car dès le lendemain, 26 avril, un réservoir d'huile à fournaise pour le smelter, d'une capacité de 4,500,000 gallons et appartenant à la Gaspe Copper, était dynamité à Mont-Louis, village situé sur la 
côte, à une cinquantaine de milles au nord de Murdochville; il se perd ainsi un million de gallons d'une valeur de $\$ 150000$. Les policiers provinciaux, au nombre d'une centaine maintenant et aidés d'une quarantaine de détectives privés à la solde de la compagnie, forcent aussitôt les mineurs à quitter leur ligne de piquetage et, en pleine nuit, les camps de la compagnie.

Le ler mai, M. Claude Jodoin, président du Congrès du travail du Canada, prie le premier ministre de la Province de convoquer au plus tôt une réunion des représentants de la compagnie et du Congrès. «Il est évident, selon lui, que tout le problème repose sur le fait que la compagnie a pris tous les moyens pour empêcher la CRO de fonctionner comme le voudraient le gouvernement et la législature... » Deux jours plus tard, M. Duplessis déclare «qu'il était tenu au courant de tout développement dans la grève des mineurs » (Le Devoir, 4 mai 1957). Le 7 mai, M. Gérard Picard, président de la CTCC, accorde l'entier appui de son groupement aux mineurs de Murdochville.

Dans une lettre datée du 20 mai, adressée à chacun des employés et lue à la radio, M. W. G. Brissenden, gérant de la compagnie, déclare que «ceux qui ne sont pas revenus à l'ouvrage ne seront plus considérés comme des employés de Gaspe Copper... Les Steelworkers ont plusieurs fois tenté d'imposer leur volonté à l'industrie minière. Cette grève n'en est qu'une parmi plusieurs autres montées par cette union (nord ontarien et nord-ouest québecois)... Les employés et leurs familles ont souffert de grandes privations durant les grèves et les employés n'ọnt rien gagné de plus qu'ils auraient eu sans grève... La compagnie se réservera toujours le droit d'engager et le droit de garder les employés qu'elle engagera... Cette grève ne sera pas réglé (sic!) par aucune entente entre la Compagnie et les métallurgistes unis ».

Du 22 mai au 6 juin, quelques échauffourées se produisent entre grévistes, non-grévistes et policiers, et le piquetage des six hommes qui avaient reçu autorisation le 2 mai est de nouveau interdit le 4 juin. Le 7 juin, la compagnie annonce une augmentation, horaire de 5 cents, en vigueur le ler juin, un congé statutaire de plus (Noël) avec travail à temps double, la prolongation de la durée des vacances payées, la semaine de 48 heures pour tous les départements, ainsi que la possibilité d'établir un plan d'assurance en cas de maladie ou d'accident, dont le coût serait défrayé à parts égales par la compagnie et le syndicat et qui donnerait au travailleur malade ou accidenté $\$ 30$ par semaine durant au plus 13 semaines. La compagnie changeait ainsi, de son propre chef, les conditions de travail existantes.

Le 24 juin, une légère explosion de dynamite secoue l'abri du convoyeur qui transporte le minerai du sous-sol au concentrateur et sert en même temps de poste de contrôle, sur la propriété de la compagnie; un ouvrier est blessé légèrement et la production, qui a repris partiellement, est interrompue pour un temps. Selon la compagnie, "plus de 460 employés se présentent chaque matin au travail. On aura bientôt besoin d'un plus grand nombre d'employés. Vu que de nombreux Gaspésiens ont refusé de retourner au travail, la compagnie doit maintenant lancer une campagne de recrutement de personnel pour son usine de Murdochville » ( L'Action Catholique, 28 juin 1957).

Le 27 du même mois, la Fédération des travailleurs du Québec s'en prend à une lettre du 19 juin du ministre provincial du Travail à la municipalité de SteThérèse-de-Gaspé, dans laquelle ce dernier déclare que l'intervention gouvernementale est impossible parce que la grève des. ouvriers est «illégale », ces derniers ayant décidé de quitter le travail sans attendre l'issue des procédures judiciaires amorcées par la compagnie contre la CRO. A cause de cela, dit-il, «je ne peux obliger les deux parties en cause à se rencontrer ». Ainsi s'exprime la Fédération sur cette attitude:

Il nous. semble étrange que le ministre, au lieu de soumettre le cas au procureur général afin de faire déclarer cette grève illégale par les tribunaux, s'il la croit illégale, prenne sur lui de rendre un jugement. 
Nul gouvernement à notre avis ne peut s'arroger les pouvoirs législatifs et judiciaires à la fois. Il serait plus sage de permettre aux tribunaux de décider de la légalité de cette grève. Nous pourrions établir alors, une fois pour toutes, si l'exercice du droit de grève découle du Code pénal ou des décisions ministérielles. Dans l'intervalle, le ministre du Travail pourrait s'en reporter à la Loi des différends ouvriers, prendre connaissance du conflit qui existe à la Gaspé Copper et faire des efforts pour le régler... Aucune déclaration n'a été faite par le ministre du Travail ou par le procureur général depuis le début quant à l'illégalité dans laquelle la compagnie s'est placée en prenant un bref de prohibition contre la Commission de relations ouvrières en dépit de l'article 4la de la Loi des relations ouvrières... Le ministre du Travail pourrait-il nous indiquer de quelle autre façon auraient pu agir les ouvriers à cause de l'action illégale de la compagnie? Devraient-ils se résigner à voir l'exercice de leur droit d'association enterré dans les dossiers poussiéreux de procédures soi-disant légales poursuivies à l'infini par une compagnie antisyndicale?...

Le 2 juillet, la compagnie avise ses locataires-grévistes que les loyers en souffrance depuis le début de la grève devront être pleinement acquittés dans la semaine suivante. Elle agit de même, le 8, à l'égard des paiements hypothécaires. On sait que quelque 200 grévistes habitent alors Murdochville. Le 14 juillet meurt Hervé Bernatchez, victime d'une explosion de dynamite survenue deux jours plus tôt, dans des circonstances mystérieuses, sur les terrains de la compagnie, en présence de deux camarades également grévistes. Le ler août, le gérant, $M$. Brissenden, déclare que des lettres ont été adressées aux grévistes les avisant que «s'ils ne se sont pas présentés au travail vendredi soir (le lendemain), leurs noms seront rayés de la liste de paie. En aucune circonstance nous mettrons à pied les ouvriers actuellement au travail pour les remplacer par les grévistes qui reviendront $\gg$.

Le 19 août, c'est la désormais célèbre «marche sur Murdochville », à laquelle participent, en caravane routière, quelque 450 ouvriers et leurs chefs (y compris MM. Jodoin et Picard, respectivement présidents du CTC et de la CTCC) venus de nombreux centres industriels de la province. La police leur permet de faire du piquetage paisible; ils n'en sont pas moins lapidés par les non-grévistes, dont certains sont des immigrants. Une fois qu'ils sont partis, une centaine de «voyous», dit la Presse Canadienne, saccagent les locaux du syndicat, en détruisent le mobilier et renversent une douzaine d'automobiles. Le lendemain, M. Roger Provost, président de la Fédération des travailleurs du Québec, déclare: "Le piquetage est devenu insuffisant sous le présent régime. Il faudra passer à l'action politique directe 》. Le 22 août, il accuse la police provinciale, le gouvernement et la compagnie d'avoir été de connivence directe pour organiser les actes de violence du 19. Le 25 août a lieu la manifestation ouvrière de Drummondville, où les orateurs parlent ouvertement de la nécessité d'un engagement politique vigoureux. Le 28 , le premier ministre du pays refuse d'agir dans le cas de Murdochville, pour des motifs constitutionnels. Celui du Québec estime le même jour que « les esprits sont trop échauffés et qu'il n'y a aucune chance de coopération en ce moment », ce qui expliquerait son refus persistant d'intervenir.

Le 2 septembre 1957. lors de la fête du Travail, S.Exc. Mgr Maurice Roy, s'adressant à des syndiqués catholiques de la région de Québec, déclare que

« Ces différends... deviennent particulièrement douloureux quand la vie même du syndicat est compromise et que le droit d'association est mis en jeu. Nous avons vu malheureusement, ici même et ailleurs, ce droit fondamental combattu par certains employeurs. »

Samedi, le 7 septembre, a lieu la «marche sur Québec », alors que plus de $\$ 5,000$ travailleurs de tous les coins de la Province se massent devant les édifices du Parlement pour protester contre la décision du gouvernement provincial de ne pas 
intervenir à Murdochville, et pour attirer l'attention du public sur le droit d'association des travailleurs et les problèmes ouvriers. Tout s'y passe dans l'ordre.

Le 13, 200 «émeutiers 》non identifiés s'emparent à Montréal du Mont Royal, bateau de faible tonnage transportant 350 tonnes de cuivre extrait de Murdochville. «Le cuivre de Gaspé est du cuivre de scab!», dit une pancarte laissée sur les lieux. Un homme est légèrement blessé, et le bateau de 144 tonneaux est mis hors de service pour une semaine.

Les journaux du 14 septembre parlent de la possibilité d'une grève symbolique d'une journée, en rotation, dans les principaux centres industriels de la Province, pour protester contre l'inaction du gouvernement et l'attitude antisyndicale de la Gaspe Copper Mines. Le 19, à Chandler ( 130 milles de Murdochville et 30 milles de Percé), une foule de quelque 300 personnes réussit pendant trois heures à immobiliser un convoi du CNR, dont trois wagons sont bondés d'anodes de cuivre en provenance de Murdochville.

Le 24 septembre, le juge William Morin rend jugement sur la question du bref de prohibition demandé par la compagnie contre la CRO un an plus tôt. Il décide que la CRO n'a pas excédé sa juridiction. Voici quelques passages de ce jugement, dont on trouvera le texte complet dans la section \& Jurisprudence du travail » de ce numéro de Relations Industrielles:

L'absence ou l'excès de juridiction chez les tribunaux inférieurs et chez les organismes gouvernementaux de la nature de la Commission de relations ouvrières ouvrent la porte, et cela nonobstant des dispositions légales comme celles de l'article 41 la de la Loi des relations ouvrières, à tous les recours de droit reconnus aux justiciables par la loi, dans pareils cas, pour garantir leurs libertés les plus précieusement fondamentales, recours soit par l'action directe en nullité, soit par le bref de prohibition. soit encore par le bref de certiorari.

Admettre dans loccurrence que l'article la de la Loi des relations ouvrières doit s'appliquer rigoureusement et au strict pied de la lettre de son texte dans tous les cas, même dans les cas d'absence totale d'excès ou d'abus pure et simple de juridiction de la part d'organismes administratifs régis par cette loi rendant des décisions ou ondronnances d'une nature judiciaire ou à peu près judiciaire équivaudrait à taxer le législateur d'avoir voulu en sous-main et pharisaïquement restreindre et diminuer les libertés constitutionnelles et fondamentales du citoyen et les recours du justiciable aux tribunaux supérieurs.

L'article 4la de la Loi des relations ouvrières ne saurait donc s'appliquer malgré toute la clarté et toute la rigueur de son texte suppressif que dans le cas et uniquement dans le cas où l'intimée, la Commission de relations ouvrières, n'aurait aucunement excédé les limites de la juridiction qui lui est assignée par la Loi des relations ouvrières.

Il ne semble y avoir dans tout ce litige aucun procédé de la part de l'intimée (CRO) à l'égard de la requérante (Gaspe Copper) pouvant s'interpréter de près ou de loin comme un déni de justice, comme un refus d'audition et encore moins comme une manoeuvre de la part de l'intimée (CRO) pour entourer la mise en cause (United Steelworkers of America) de mystère et d'impalpabilité...

Que l'intimée (CRO) ait jugé préférable de ne pas fournir les noms des officiers ni une copie de tout son dossier ou encore ait fourni des documents trouvés informes par la requérante (Gaspe Copper Mines), ca peut être un procédé plus ou moins recommandable du strict point de vue de la courtoisie et de l'étiquette professionnelle, mais de là à invoquer... un déni de justice, un refus d'audition, une violation flagrante de tous les usages et de toutes les règles de nos procédures judiciaires et une violation du droit naturel et constitutionnel de toute partie à un 
litige ainsi qu'un excès de juridiction, il y a toute une marge en droit dont le justiciable doit tenir compte surtout, encore une fois, lorsqu'il s'agit pour lui de recourir à des procédures de l'importance des brefs de prérogatives...

Le Tribunal ne voit rien dans la Loi des Relations Ouvrières obligeant l'intimée (CRO) à l'accomplissement de formalités particulières relatives aux renseignements à donner aux intéressés lors du dépôt d'une requête en reconnaissance syndicale...

Une longue parenthèse s'impose ici pour expliquer pourquoi il a fallu près de quatorze mois à un juge de la Cour Supérieure pour rendre jugement dans une affaire dont les répercussions socio-économiques allaient augmentant à un rythme rapide à mesure que passaient les jours.

Trois semaines après l'émission du bref du 2 août 1956, le procureur de la CRO remplit la formalité prévue de la comparution par écrit, la Cour se trouvant ajournée jusqu'au 10 septembre suivant, comme c'est la coutume. Ce jour-là même, ledit procureur soumet une inscription en droit totale pour demander le renvoi du bref, s'appuyant sur l'article 41 a de la Loi des relations ouvrières, qui dit que «a) les décisions de la Commission sont sans appel et ne peuvent être revisées par les tribunaux; et b) aucun bref... de prohibition... ne peut être émis contre la Commission... ». Subsidiairement, la CRO allègue que, même en l'absence de l'article 4la, elle n’a pas excédé sa juridiction.

Pendant les deux semaines qui suivent, les procureurs de la CRO et du syndicat préparent leur plaidoirie sur l'inscription et se présentent devant un juge qui, surchargé d'ouvrage, se récuse. Un mois plus tard, soit le 25 octobre 1956, un nouveau juge (Morin) propose aux procureurs des parties - ou ce sont les procureurs qui demandent au juge - que les plaidoyers se fassent plutôt par écrit (factum), à trois semaines de là; on s'accorde sur ce point. Le procureur du syndicat, qui avait produit une comparution dans l'action, s'en remet à son collègue de la CRO pour la préparation dudit factum, qui est confié à la poste le 2 novembre suivant. Le 8 du même mois, l'affaire Transport Boischatel Ltée, qui à l'instar d'une autre cause alors en suspens (Syndicat national des employés de la pulpe et du papier de La Tuque vs CRO) comporte maintes analogies avec celle de la Gaspe Copper, est transférée devant sept juges de la Cour d'Appel pour qu'ils tranchent une fois pour toutes, à la demande expresse du juge en chef, la question de la validité de l'article 41 a tel qu'amendé en 1953. Le juge Morin estima alors préférable d'attendre l'arrêt des sept juges pour se prononcer, ou encore accéda aux désirs des procureurs de la CRO et de la Gaspe Copper; quoi qu'il en soit, c'est ainsi qu'il fut fait.

L'affaire Transport Boischatel Ltée ne fut entendue qu'en février, et ce n'est que six mois plus tard, soit le ler août, que les sept juges prononçèrent leur arrêt en faveur de la CRO, estimant que celle-ci n'avait pas excédé sa juridiction, mais sans se prononcer directement sur la question de l'article 41 a de la Loi des relations ouvrières. (Cf. texte intégral dans section Jurisprudence du travail de ce numéro.) On avait donc attendu en vain tous ces longs mois. Le même jour, le juge demande au procureur de la Gaspe Copper de présenter son factum; celui-ci s'exécute le 10 septembre dernier. Et le jugement sort le 21 septembre, dans le sens indiqué plus haut.

Enfin, le 5 octobre au soir, 300 grévistes réunis au sous-sol de l'église de Murdochville décident, à $80 \%$ près, de rentrer au travail le lundi suivant, 7 octobre. Maís la compagnie déclare immédiatement n'en avoir besoin que de 200 , et encore, pas immédiatement; car, après l'ultimatum d'août, elle entend garder 800 nongrévistes.

Et voilà, pour un sec bilan de «l'affaire de Murdochville ». 